MATHEMATICS OF COMPUTATION

Volume 73, Number 247, Pages 1325-1343

S 0025-5718(03)01587-4

Article electronically published on July 28, 2003

\title{
SINC-GALERKIN METHOD FOR SOLVING LINEAR SIXTH-ORDER BOUNDARY-VALUE PROBLEMS
}

\author{
MOHAMED EL-GAMEL, JOHN R. CANNON, AND AHMED I. ZAYED
}

\begin{abstract}
There are few techniques available to numerically solve sixth-order boundary-value problems with two-point boundary conditions. In this paper we show that the Sinc-Galerkin method is a very effective tool in numerically solving such problems. The method is then tested on examples with homogeneous and nonhomogeneous boundary conditions and a comparison with the modified decomposition method is made. It is shown that the Sinc-Galerkin method yields better results.
\end{abstract}

\section{INTRODUCTION}

Sixth-order boundary-value problems (BVPs) are known to arise in astrophysics; the narrow convecting layers bounded by stable layers, which are believed to surround A-type stars, may be modelled by sixth-order BVPs [3, 16]. Further discussion of the sixth-order BVPs are given in [2].

The literature of numerical analysis contains little on the solution of the sixthorder BVPs [3, 13, 16]. Theorems that list conditions for the existence and uniqueness of solutions of such problems are thoroughly discussed in 1], but no numerical methods are contained therein.

In [2] nonnumerical techniques were developed for solving such BVPs, but numerical methods of solutions were introduced implicity by Chawla [4. Recently, in [17, the Adomain decomposition method and modified decomposition method were used to investigate sixth-order boundary-value problems by Wazwaz.

The present work describes a Sinc-Galerkin method for the solution of sixth-order ordinary differential equations of the form

$$
L u(x)=u^{(6)}+\sum_{k=0}^{5} p_{k}(x) u^{(k)}(x)=f(x), \quad 0 \leq x \leq 1,
$$

where $p_{k}(x)$ are analytic and satisfy some extra conditions to be stated later (see Theorems 2.3 and 2.4), subject to boundary conditions

$$
u^{(i)}(0)=0, \quad u^{(i)}(1)=0, \quad i=0,1,2 .
$$

The Sinc-Galerkin method utilizes a modified Galerkin scheme to discretize (1.1). The basis elements that are used in this approach are the Sinc functions composed

Received by the editor June 27, 2002 and, in revised form, December 10, 2002.

2000 Mathematics Subject Classification. Primary 65L60; Secondary 65L10.

Key words and phrases. Sinc functions, Sinc-Galerkin method, sixth-order differential equations, numerical solutions.

(C)2003 American Mathematical Society 
with a suitable conformal map. A thorough description of the Sinc-Galerkin method may be found in 9 .

The Sinc-Galerkin method for ordinary differential equations has many salient problem features due to the properties of the basis functions and the manner in which the problem is discretized. Of equal practical significance is the fact that the method's implementation requires no modification in the presence of singularities. There are several schemes of the Sinc-Galerkin method; the one we shall use is known as the approximation of the derivatives method.

The paper is organized into four sections. Section 2 outlines the known Sinc properties that are necessary for the formulation of the discrete linear system. The Sinc-Galerkin approximation, as well as the choice of the inner product, and error bounds for the approximation are presented. The remainder of the section is devoted to the derivation of the discrete system. Section 3 presents appropriate techniques to treat nonhomogenous boundary conditions. Section 4 is devoted to examples. We have selected three examples. The first one is on a homogenous problem and the last two are on nonhomogenous problems. The examples show the numerical performance of the Sinc-Galerkin method and demonstrate its ability to deal with nonhomogenous boundary conditions.

\section{The Sinc-Galerkin method}

The Sinc-Galerkin procedure for the problem in equations (1.1)-(1.2) begins by selecting composite Sinc functions appropriate to the interval $(0,1)$ as the basis functions. Definitions, notation, and properties of Sinc functions and composite Sinc functions can be found in references [5, 8, 9, 14]. The appropriate composite Sinc functions, $S(j, h) \circ \phi(x)$, over the interval $x \in(0,1)$ are defined as

$$
\begin{aligned}
S_{j}(x) & =S(j, h) \circ \phi(x) \\
& =\operatorname{Sinc}\left(\frac{\phi(x)-j h}{h}\right), \quad j \text { is an integer }
\end{aligned}
$$

where the conformal map is given by

$$
\phi(x)=\ln \left(\frac{x}{1-x}\right)
$$

which carries the domain $D_{E}$,

$$
D_{E}=\left\{z=x+i y:\left|\arg \left(\frac{z}{1-z}\right)\right|<d \leq \frac{\pi}{2}\right\},
$$

onto the infinite strip $D_{d}$,

$$
D_{d}=\left\{w=u+i v:|v|<d \leq \frac{\pi}{2}\right\} .
$$

The approximate solution for $u(x)$ is represented by the formula

$$
u_{m}(x)=\sum_{j=-M}^{N} c_{j} S_{j}(x), \quad m=M+N+1 .
$$

The unknown coefficients $c_{j}$ in equation (2.5) are determined by orthogonalizing the residual with respect to the basis functions, i.e.,

$$
\left\langle L u_{m}-f, S_{k}\right\rangle=0, \quad-M \leq k \leq N .
$$


The choice of the inner product that is used in equation (2.6), along with the choice of basis functions, determines the properties of the approximation method. The inner product that is used for the Sinc-Galerkin method is defined by

$$
\langle f, g\rangle=\int_{0}^{1} f(x) g(x) w(x) d x .
$$

Here $w(x)$ is a weight function that is chosen depending on the boundary conditions, the domain, and the differential equation. For the case of sixth-order problems, it is convenient to take

$$
w(x)=\frac{1}{\left(\phi^{\prime}(x)\right)^{3}} .
$$

A complete discussion of the choice of the weight function can be found in 914 15.

The most direct development of the discrete system for (1.1) is obtained by substituting (2.5) into (2.6). This approach, however, obscures the analysis that is necessary for applying the Sinc quadrature formulas to (2.6). An alternative approach is to analyze instead

$$
\left\langle\sum_{j=0}^{6} p_{j}(x) u^{(j)}(x), S_{k}\right\rangle=\left\langle f, S_{k}\right\rangle, p_{6}(x)=1 ; \quad-M \leq k \leq N .
$$

The integrals in (2.9) are approximated by the Sinc quadrature rule [9, 10]. To describe this quadrature rule, we need the following definition and theorems:

Definition 1. Let $D_{E}$ be a simply connected domain in the complex plane $\mathbb{C}$, and let $\partial D_{E}$ denote the boundary of $D_{E}$. Let $a, b(a \neq b)$ be points on $\partial D$, and let $\phi$ be a conformal mapping that maps $D_{E}$ onto $D_{d}$ such that $\phi(a)=-\infty$ and $\phi(b)=\infty$. If the inverse map of $\phi$ is denoted by $\psi$, define

$$
\Gamma=\{\psi(u):-\infty<u<\infty\}
$$

and

$$
z_{k}=\psi(k h), \quad k=0, \pm 1, \pm 2, \ldots
$$

Definition 2. Let $B\left(D_{E}\right)$ be the class of functions $F$ that are analytic in $D_{E}$ and that satisfy

$$
\int_{\psi(\gamma+u)}|F(z) d z| \rightarrow 0, \quad \text { as } u \rightarrow \pm \infty
$$

where

$$
\gamma=\left\{i y:|y|<d \leq \frac{\pi}{2}\right\}
$$

and that satisfy

$$
T(F)=\int_{\partial D_{E}}|F(z) d z|<\infty,
$$

on the boundary of $D_{E}$ (denoted $\left.\partial D_{E}\right)$.

The following theorem for functions in $B\left(D_{E}\right)$ can be found in [14].

Theorem 2.1. Let $\Gamma$ be $(0,1)$. If $F \in B\left(D_{E}\right)$ and

$$
x_{j}=\phi^{-1}(j h)=\psi(j h)=\frac{e^{j h}}{1+e^{j h}}, \quad j=0, \pm 1, \pm 2, \ldots,
$$


then for $h>0$ sufficiently small

$$
\int_{\Gamma} F(x) d x-h \sum_{j=-\infty}^{\infty} \frac{F\left(x_{j}\right)}{\phi^{\prime}\left(x_{j}\right)}=\frac{i}{2} \int_{\partial D} \frac{F(z) k(\phi, h)(z)}{\sin (\pi \phi(z) / h)} d z \equiv I_{F},
$$

where

$$
\left.|k(\phi, h)|\right|_{z \in \partial D}=\left|\exp \left[\frac{i \pi \phi(z)}{h} \operatorname{sgn}(\operatorname{Im}(\phi(z)))\right]\right|_{z \in \partial D}=e^{-\pi d / h} .
$$

For the Sinc-Galerkin method, the infinite quadrature rule must be truncated to a finite sum. The following theorem indicates the conditions under which exponential convergence results.

Theorem 2.2. If there exist positive constants $\alpha, \beta$ and $C$ such that

$$
\left|\frac{F(x)}{\phi^{\prime}(x)}\right| \leq C \begin{cases}\exp (-\alpha|\phi(x)|), & x \in(-\infty, 0), \\ \exp (-\beta|\phi(x)|), & x \in[0, \infty) .\end{cases}
$$

Then the error bound for the quadrature rule (2.14) is

$$
\left|\int_{0}^{1} F(x) d x-h \sum_{j=-M}^{N} \frac{F\left(x_{j}\right)}{\phi^{\prime}\left(x_{j}\right)}\right| \leq C\left(\frac{e^{-\alpha M h}}{\alpha}+\frac{e^{-\beta N h}}{\beta}\right)+\left|I_{F}\right| .
$$

The infinite sum in (2.14) is truncated and with the use of (2.16) we arrive at the inequality (2.17). Making the selections

$$
h=\sqrt{\frac{\pi d}{\alpha M}}
$$

and

$$
N \equiv\left[\left|\frac{\alpha}{\beta} M+1\right|\right]
$$

where $[x]$ is the integer part of $x$, it will follow that if we can show that $\left|I_{F}\right| \leq$ $C e^{-\pi d / h}$, then equation (2.17) will yield,

$$
\int_{0}^{1} F(x) d x=h \sum_{j=-M}^{N} \frac{F\left(x_{j}\right)}{\phi^{\prime}\left(x_{j}\right)}+O\left(e^{-(\pi \alpha d M)^{1 / 2}}\right) .
$$

The Sinc quadrature rule is the replacement of the integral on the left-hand side of (2.20) by the sum on the right-hand side. Most of the remainder of this section is devoted to the derivation of the estimates of $I_{F}$, which validate the use of formula (2.20) in the integrals associated with the sixth-order Sinc-Galerkin method.

In the application of the truncated quadrature rule (2.20) to the integrals in (2.9), we need to evaluate

$$
\delta_{j k}^{(P)}=h^{P}\left[\frac{d^{P}}{d \phi^{P}}[S(j, h) \circ \phi(x)]\right]_{x=x_{k}}, \quad 0 \leq P \leq 6 .
$$


It is easy to verify that

$$
\begin{aligned}
& \delta_{j k}^{(0)}=\left.[S(j, h) \circ \phi(x)]\right|_{x=x_{k}}= \begin{cases}1, & j=k, \\
0, & j \neq k,\end{cases} \\
& \delta_{j k}^{(1)}=\left.h \frac{d}{d \phi}[S(j, h) \circ \phi(x)]\right|_{x=x_{k}}=\left\{\begin{array}{l}
0, \quad j=k, \\
\frac{(-1)^{k-j}}{k-j}, \quad j \neq k,
\end{array}\right. \\
& \delta_{j k}^{(2)}=\left.h^{2} \frac{d^{2}}{d \phi^{2}}[S(j, h) \circ \phi(x)]\right|_{x=x_{k}}=\left\{\begin{array}{l}
\frac{-\pi^{2}}{3}, \quad j=k, \\
\frac{-2(-1)^{k-j}}{(k-j)^{2}}, \quad j \neq k .
\end{array}\right.
\end{aligned}
$$

The following quantities were evaluated in [12] to solve fourth-order problems:

$$
\delta_{j k}^{(3)}=\left.h^{3} \frac{d^{3}}{d \phi^{3}}[S(j, h) \circ \phi(x)]\right|_{x=x_{k}}= \begin{cases}0, & j=k, \\ \frac{(-1)^{k-j}}{(k-j)^{3}}\left[6-\pi^{2}(k-j)^{2}\right], & j \neq k,\end{cases}
$$

$$
\delta_{j k}^{(4)}=\left.h^{4} \frac{d^{4}}{d \phi^{4}}[S(j, h) \circ \phi(x)]\right|_{x=x_{k}}= \begin{cases}\frac{\pi^{4}}{5}, & j=k, \\ \frac{-4(-1)^{k-j}}{(k-j)^{4}}\left[6-\pi^{2}(k-j)^{2}\right], & j \neq k .\end{cases}
$$

To solve the sixth-order equation (1.1)-(1.2), we need the following lemma whose proof is straightforward and will be left to the reader.

Lemma 2.1. Let $\phi$ be the conformal one-to-one mapping of the simply connected domain $D_{E}$ onto $D_{d}$, given by (2.2). Then

$$
\delta_{j k}^{(5)}=\left.h^{5} \frac{d^{5}}{d \phi^{5}}[S(j, h) \circ \phi(x)]\right|_{x=x_{k}}= \begin{cases}0, & j=k, \\ \kappa_{j k}, & j \neq k,\end{cases}
$$

where

$$
\begin{aligned}
& \kappa_{j k}=\frac{(-1)^{k-j}}{(k-j)^{5}}\left[120-20 \pi^{2}(k-j)^{2}+\pi^{4}(k-j)^{4}\right], \\
& \delta_{j k}^{(6)}=\left.h^{6} \frac{d^{6}}{d \phi^{6}}[S(j, h) \circ \phi(x)]\right|_{x=x_{k}}= \begin{cases}\frac{\pi^{6}}{7}, & j=k, \\
\mu_{j k}, & j \neq k,\end{cases}
\end{aligned}
$$

where

$$
\mu_{j k}=\frac{-6(-1)^{k-j}}{(k-j)^{6}}\left[120-20 \pi^{2}(k-j)^{2}+\pi^{4}(k-j)^{4}\right],
$$

The method of approximating the integrals in (2.9) begins by integrating by parts to transfer all derivatives from $u$ to $S_{k}$. The approximation of the last five inner products on the right-hand side of (2.9) has been thoroughly treated in 12 . We will list them for convenience

$$
\left\langle p_{3} u^{(3)}, S_{k}\right\rangle=-h \sum_{j=-\infty}^{\infty} \sum_{i=0}^{3} \frac{u\left(x_{j}\right)}{\phi^{\prime}\left(x_{j}\right) h^{i}} \delta_{k j}^{(i)} g_{3, i}-I_{F}^{(3)},
$$


where

$$
\begin{gathered}
g_{3,3}=\left(p_{3} w\right)\left(\phi^{\prime}\right)^{3}, \\
g_{3,2}=3\left(p_{3} w\right) \phi^{\prime} \phi^{\prime \prime}+3\left(p_{3} w\right)^{\prime}\left(\phi^{\prime}\right)^{2}, \\
g_{3,1}=\left(p_{3} w\right) \phi^{(3)}+3\left(p_{3} w\right)^{\prime} \phi^{(2)}+3\left(p_{3} w\right)^{\prime \prime} \phi^{\prime},
\end{gathered}
$$

and

$$
\begin{gathered}
g_{3,0}=\left(p_{3} w\right)^{(3)} \\
\left\langle p_{2} u^{(2)}, S_{k}\right\rangle=h \sum_{j=-\infty}^{\infty} \sum_{i=0}^{2} \frac{u\left(x_{j}\right)}{\phi^{\prime}\left(x_{j}\right) h^{i}} \delta_{k j}^{(i)} g_{2, i}+I_{F}^{(2)}
\end{gathered}
$$

where

$$
\begin{gathered}
g_{2,2}=\left(p_{2} w\right)\left(\phi^{\prime}\right)^{2}, \\
g_{2,1}=\left(p_{2} w\right) \phi^{\prime \prime}+2\left(p_{2} w\right)^{\prime} \phi^{\prime},
\end{gathered}
$$

and

$$
g_{2,0}=\left(p_{2} w\right)^{\prime \prime}
$$

and finally

$$
\left\langle p_{1} u^{(1)}, S_{k}\right\rangle=-h \sum_{j=-\infty}^{\infty} \sum_{i=0}^{1} \frac{u\left(x_{j}\right)}{\phi^{\prime}\left(x_{j}\right) h^{i}} \delta_{k j}^{(i)} g_{1, i}-I_{F}^{(1)},
$$

where

$$
\begin{gathered}
g_{1,1}=\left(p_{1} w\right) \phi^{\prime}, \\
g_{1,0}=\left(p_{1} w\right)^{\prime},
\end{gathered}
$$

and

$$
\left\langle G, S_{k}\right\rangle=h \frac{G\left(x_{k}\right) w\left(x_{k}\right)}{\phi^{\prime}\left(x_{k}\right)}+I_{F}^{(0)} .
$$

The integrals $I_{F}^{(3)}, I_{F}^{(2)}$, and $I_{F}^{(1)}$ are the contour integral errors on the right-hand side of (2.14) with $F$ replaced by $u\left(p_{3} S_{k} w\right)^{\prime \prime \prime}, u\left(p_{2} S_{k} w\right)^{\prime \prime}$, and $u\left(p_{1} S_{k} w\right)^{\prime}$, respectively. Lastly, (2.40) holds where $G$ is either $p_{0} u$ or $f$. When $G$ is $p_{0} u$ or $f, I_{F}^{(0)}$ is the contour integral error in (2.14) with $F$ replaced by $u\left(p_{0} S_{k} w\right)$ or $f\left(S_{k} w\right)$, respectively.

To solve the equation (1.1)-(1.2), we need the following theorem

Theorem 2.3. The following relations hold:

$$
\begin{aligned}
\left\langle u^{(6)}, S_{k}\right\rangle & =h \sum_{j=-\infty}^{\infty} \sum_{i=0}^{6} \frac{u\left(x_{j}\right)}{\phi^{\prime}\left(x_{j}\right) h^{i}} \delta_{k j}^{(i)} g_{6, i}+I_{F}^{(6)}, \\
\left\langle p_{5} u^{(5)}, S_{k}\right\rangle & =-h \sum_{j=-\infty}^{\infty} \sum_{i=0}^{5} \frac{u\left(x_{j}\right)}{\phi^{\prime}\left(x_{j}\right) h^{i}} \delta_{k j}^{(i)} g_{5, i}-I_{F}^{(5)},
\end{aligned}
$$

and

$$
\left\langle p_{4} u^{(4)}, S_{k}\right\rangle=h \sum_{j=-\infty}^{\infty} \sum_{i=0}^{4} \frac{u\left(x_{j}\right)}{\phi^{\prime}\left(x_{j}\right) h^{i}} \delta_{k j}^{(i)} g_{4, i}+I_{F}^{(4)}
$$


for some functions $g_{i, j}$ to be determined, where $I_{F}^{(6)}, I_{F}^{(5)}$ and $I_{F}^{(4)}$ are the contour integral errors on the right-hand side of (2.14) with $F$ replaced by $u\left(S_{k} w\right)^{(6)}$, $u\left(p_{5} S_{k} w\right)^{(5)}$ and $u\left(p_{4} S_{k} w\right)^{(4)}$, respectively.

Proof. For $u^{(6)}$, the inner product with Sinc basis elements is given by

$$
\left\langle u^{(6)}, S_{k}\right\rangle=\int_{0}^{1} u^{(6)} S_{k}(x) w(x) d x .
$$

Integrating by parts to remove the sixth derivatives from the dependent variable $u$ leads to the equality

$$
\int_{0}^{1} u^{(6)}(x) S_{k}(x) w(x) d x=B_{T, 6}+\int_{0}^{1} u(x)\left(S_{k}(x) w(x)\right)^{(6)} d x,
$$

where the boundary term

$$
B_{T, 6}=\left[\sum_{i=0}^{5}(-1)^{i} u^{(5-i)}\left(S_{k} w\right)^{(i)}\right]_{x=0}^{1}
$$

is zero because the first three terms vanish due to the fact that $w=w^{\prime}=w^{\prime \prime}=0$ at $x=0,1$ and the last three terms vanish due to the fact that $u$ satisfies the boundary conditions (1.2). Setting

$$
\frac{d^{n}}{d \phi^{n}}\left[S_{k}(x)\right]=S_{k}^{(n)}(x), \quad 0 \leq n \leq 6,
$$

and noting that

$$
\frac{d}{d x}\left[S_{k}(x)\right]=S_{k}^{(1)}(x) \phi^{\prime}(x),
$$

by expanding the derivatives under the integral in (2.44) we obtain

$$
\left\langle u^{(6)}, S_{k}(x)\right\rangle=\int_{0}^{1}\left(\sum_{i=0}^{6} u(x) S_{k}^{(i)}(x) g_{6, i}\right) d x,
$$

where

$$
\begin{gathered}
g_{6,6}=w\left(\phi^{\prime}\right)^{6} \\
g_{6,5}=15 w\left(\phi^{\prime}\right)^{4} \phi^{\prime \prime}+6 w^{\prime}\left(\phi^{\prime}\right)^{5} \\
g_{6,4}=20 w \phi^{(3)}\left(\phi^{\prime}\right)^{(3)}+45 w\left(\phi^{\prime}\right)^{2}\left(\phi^{\prime \prime}\right)^{2}+60 w^{\prime}\left(\phi^{\prime}\right)^{3} \phi^{\prime \prime}+15 w^{\prime \prime}\left(\phi^{\prime}\right)^{(4)} \\
g_{6,3}=15 w\left(\phi^{\prime \prime}\right)^{3}+15 w\left(\phi^{\prime}\right)^{2}(\phi)^{(4)}+60 w \phi^{\prime} \phi^{\prime \prime} \phi^{\prime \prime \prime} \\
+60 w^{\prime}\left(\phi^{\prime}\right)^{2} \phi^{\prime \prime \prime}+90 w^{\prime} \phi^{\prime}\left(\phi^{\prime \prime}\right)^{2}+90 w^{\prime \prime} \phi^{\prime \prime}\left(\phi^{\prime}\right)^{2}+20 w^{\prime \prime \prime}\left(\phi^{\prime}\right)^{3}, \\
g_{6,2}=10 w\left(\phi^{\prime \prime \prime}\right)^{2}+6 w \phi^{\prime} \phi^{(5)}+15 w \phi^{\prime \prime} \phi^{(4)}+30 w^{\prime} \phi^{\prime} \phi^{(4)}+60 w^{\prime} \phi^{\prime \prime} \phi^{\prime \prime \prime} \\
+60 w^{\prime \prime} \phi^{\prime} \phi^{\prime \prime \prime}+45 w^{\prime \prime}\left(\phi^{\prime \prime}\right)^{2}+60 w^{\prime \prime \prime} \phi^{\prime} \phi^{\prime \prime}+15 w^{(4)}\left(\phi^{\prime}\right)^{2}, \\
g_{6,1}=\phi^{(6)} w+6 \phi^{(5)} w^{\prime}+15 \phi^{(4)} w^{(2)}+20 \phi^{(3)} w^{(3)}+15 \phi^{(2)} w^{(4)}+6 \phi^{\prime} w^{(5)},
\end{gathered}
$$

and

$$
g_{6,0}=w^{(6)} .
$$

Applying the Sinc quadrature rule to the right-hand side of (2.46) yields (2.41). 
The inner product for $\left\{p_{5}(x) u^{(5)}(x)\right\}$ may be handled in a similar manner to yield

$$
\int_{0}^{1} u^{(5)}(x) S_{k}(x) p_{5}(x) w(x) d x=B_{T, 5}-\int_{0}^{1} u(x)\left(S_{k}(x) p_{5} w(x)\right)^{(5)} d x,
$$

where the boundary term is

$$
B_{T, 5}=\left[\sum_{i=0}^{4}(-1)^{i} u^{(4-i)}\left(S_{k} p_{5} w\right)^{(i)}\right]_{x=0}^{1}=0 .
$$

Thus, (2.54) may be written as

$$
\left\langle p_{5}(x) u^{(5)}, S_{k}\right\rangle=-\int_{0}^{1}\left(\sum_{i=0}^{5} u(x) S_{k}^{(i)}(x) g_{5, i}\right) d x,
$$

where

$$
\begin{gathered}
g_{5,5}=p_{5} w\left(\phi^{\prime}\right)^{5} \\
g_{5,4}=10\left(p_{5} w\right)\left(\phi^{\prime}\right)^{3} \phi^{\prime \prime}+5\left(p_{5} w\right)^{\prime}\left(\phi^{\prime}\right)^{4},
\end{gathered}
$$

$$
g_{5,3}=10\left(p_{5} w\right)\left(\phi^{\prime}\right)^{2} \phi^{\prime \prime \prime}+15\left(p_{5} w\right) \phi^{\prime}\left(\phi^{\prime \prime}\right)^{2}+30\left(p_{5} w\right)^{\prime}\left(\phi^{\prime}\right)^{2} \phi^{\prime \prime}+10\left(p_{5} w\right)^{\prime \prime}\left(\phi^{\prime}\right)^{3},
$$

$$
\begin{aligned}
& g_{5,2}=5\left(p_{5} w\right) \phi^{(4)} \phi^{\prime}+10\left(p_{5} w\right) \phi^{\prime \prime} \phi^{\prime \prime \prime}+15\left(p_{5} w\right)^{\prime}\left(\phi^{\prime \prime}\right)^{2} \\
&+20\left(p_{5} w\right)^{\prime} \phi^{\prime} \phi^{\prime \prime \prime}+30\left(p_{5} w\right)^{\prime \prime} \phi^{\prime} \phi^{\prime \prime}+10\left(p_{5} w\right)^{\prime \prime \prime}\left(\phi^{\prime}\right)^{2}, \\
& g_{5,1}=\left(p_{5} w\right) \phi^{(5)}+5\left(p_{5} w\right)^{\prime} \phi^{(4)}+10\left(p_{5} w\right)^{\prime \prime} \phi^{\prime \prime \prime}+10\left(p_{5} w\right)^{\prime \prime \prime} \phi^{\prime \prime}+5\left(p_{5} w\right)^{(4)} \phi^{\prime},
\end{aligned}
$$

and

$$
g_{5,0}=\left(p_{5} w\right)^{(5)}
$$

Applying the Sinc quadrature rule to the right-hand side of (2.56) yields (2.42).

Similarly, for $\left\{p_{4}(x) u^{(4)}(x)\right\}$, after four integrations by parts to remove the four derivatives from the dependent variable $u$, we have the equality

$$
\int_{0}^{1} u^{(4)}(x) S_{k}(x) p_{4}(x) w(x) d x=B_{T, 4}+\int_{0}^{1} u(x)\left(S_{k}(x) p_{4}(x) w(x)\right)^{(4)} d x,
$$

where the boundary term is

$$
B_{T, 4}=\left[\sum_{i=0}^{3}(-1)^{i} u^{(3-i)}\left(S_{k} p_{4} w\right)^{(i)}\right]_{x=0}^{1}=0 .
$$

Then (2.63) may be written as

$$
\left\langle p_{4}(x) u^{(4)}, S_{k}\right\rangle=\int_{0}^{1}\left(\sum_{i=0}^{4} u(x) S_{k}^{(i)}(x) g_{4, i}\right) d x,
$$


where

$$
\begin{gathered}
g_{4,4}=\left(p_{4} w\right)\left(\phi^{\prime}\right)^{4}, \\
g_{4,3}=6\left(p_{4} w\right)\left(\phi^{\prime}\right)^{2} \phi^{\prime \prime}+4\left(p_{4} w\right)^{\prime}\left(\phi^{\prime}\right)^{3}, \\
g_{4,2}=3\left(p_{4} w\right)\left(\phi^{\prime \prime}\right)^{2}+4\left(p_{4} w\right) \phi^{\prime} \phi^{\prime \prime \prime}+12\left(p_{4} w\right)^{\prime} \phi^{\prime} \phi^{\prime \prime}+6\left(p_{4} w\right)^{\prime \prime}\left(\phi^{\prime}\right)^{2}, \\
g_{4,1}=\left(p_{4} w\right) \phi^{(4)}+4\left(p_{4} w\right)^{\prime} \phi^{\prime \prime \prime}+6\left(p_{4} w\right)^{\prime \prime} \phi^{\prime \prime}+4\left(p_{4} w\right)^{\prime \prime \prime} \phi^{\prime},
\end{gathered}
$$

and

$$
g_{4,0}=\left(p_{4} w\right)^{(4)} .
$$

Similarly, applying the Sinc quadrature rule to the right-hand side of (2.64) yields (2.43).

To bound the error integrals in (2.41), (2.42) and (2.43), the following lemma will be helpful.

Lemma 2.2. Let $\phi$ be as before. If $\operatorname{Im} z=d>0$ and $k$ is an integer, then for $d, h>0$,

$$
\left|\frac{\frac{d^{n}}{d \phi^{n}} S(k, h) \circ \phi(z)}{\sin \left(\frac{\pi \phi(z)}{h}\right)}\right|_{z \in \partial D_{E}} \leq C_{n}(h, d), \quad 0 \leq n \leq 6
$$

where

$$
\begin{aligned}
& C_{0}(h, d) \equiv \frac{h}{\pi d}, \quad C_{1}(h, d) \equiv \frac{1}{d} C_{0}(h, d)+\frac{1}{d \tanh \left(\frac{\pi d}{h}\right)}, \\
& C_{2}(h, d) \equiv \frac{2}{d} C_{1}(h, d)+\frac{\pi}{h d}, \quad C_{3}(h, d) \equiv \frac{3}{d} C_{2}(h, d)+\frac{\pi^{2}}{d h^{2} \tanh \left(\frac{\pi d}{h}\right)}, \\
& C_{4}(h, d) \equiv \frac{4}{d} C_{3}(h, d)+\frac{\pi^{3}}{h^{3} d}, \quad C_{5}(h, d) \equiv \frac{5}{d} C_{4}(h, d)+\frac{\pi^{4}}{d h^{4} \tanh \left(\frac{\pi d}{h}\right)},
\end{aligned}
$$

and

$$
C_{6}(h, d) \equiv \frac{6}{d} C_{5}(h, d)+\frac{\pi^{5}}{h^{5} d} .
$$

Proof. It is enough to prove the case $n=6$ in equation (2.70) Since the proofs of the remaining cases are similar. Upon noting that

$$
\left|\frac{\frac{d^{n}}{d \phi^{n}}[S(k, h) \circ \phi(z)]}{\sin (\pi \phi(z) / h)}\right|_{z \in \partial D_{E}}=\left|\frac{\frac{d^{n}}{d z^{n}}[S(k, h)(z)]}{\sin (\pi z / h)}\right|_{z=x \pm i d},
$$

we have

$$
\begin{aligned}
W=\frac{d^{6}}{d z^{6}}[S(k, h)(z)]= & \frac{-\left(\frac{\pi}{h}\right)^{5} \sin \left(\frac{\pi}{h}(z-k h)\right)}{(z-k h)} \\
& -6 \frac{\left(\frac{\pi}{h}\right)^{4} \cos \left(\frac{\pi}{h}(z-k h)\right)}{(z-k h)^{2}}+30 \frac{\left(\frac{\pi}{h}\right)^{3} \sin \left(\frac{\pi}{h}(z-k h)\right)}{(z-k h)^{3}} \\
& +120 \frac{\left(\frac{\pi}{h}\right)^{2} \cos \left(\frac{\pi}{h}(z-k h)\right)}{(z-k h)^{4}}-360 \frac{\left(\frac{\pi}{h}\right) \sin \left(\frac{\pi}{h}(z-k h)\right)}{(z-k h)^{5}} \\
& -720 \frac{\cos \left(\frac{\pi}{h}(z-k h)\right)}{(z-k h)^{6}}+720 \frac{\left(\frac{h}{\pi}\right) \sin \left(\frac{\pi}{h}(z-k h)\right)}{(z-k h)^{7}} .
\end{aligned}
$$


Now if $\operatorname{Im} z=d$, then

$$
|z-k h| \geq d, \quad\left|\cos \frac{\pi}{h}(z-k h)\right| \leq \cosh \frac{\pi d}{h}, \quad\left|\sin \left(\frac{\pi z}{h}\right)\right| \geq \sinh \frac{\pi d}{h} .
$$

Taking absolute values and using these relations leads to the bound in (2.70)

$$
\left|\frac{W}{\sin \frac{\pi z}{h}}\right| \leq \frac{\left(\frac{\pi}{h}\right)^{5}}{d}+\frac{6}{d} C_{5}(h, d) \equiv C_{6}(h, d) .
$$

The following estimates were derived in [12] and recorded here for convenience:

$$
\left|I_{F}^{(i)}\right| \leq C_{i} e^{-\pi d / h}, \quad 0 \leq i \leq 3
$$

The following lemma extends the last inequality to $i=4,5,6$.

Lemma 2.3. Let $\phi, D_{E}$ and $D_{d}$ have the same meaning as before. Assume $\phi(0)=$ $-\infty, \phi(1)=\infty$ and $x_{k}=\phi^{-1}(k h)$.

(i) If $u(S w)^{(6)}, u\left(p_{5} S w\right)^{(5)}$ and $u\left(p_{4} S w\right)^{(4)}$ are in $B\left(D_{E}\right)$, then the following estimates hold:

$$
\left|I_{F}^{(i)}\right| \leq C_{i} e^{-\pi d / h}, \quad i=4,5,6 .
$$

(ii) If there exist positive constants $\alpha, \beta$, and $k$ such that

$$
\left|\frac{F(x)}{\phi^{\prime}}\right| \leq \nu \begin{cases}\exp (-\alpha|\phi(x)|), & x \in(-\infty, 0), \\ \exp (-\beta|\phi(x)|), & x \in[0, \infty),\end{cases}
$$

where $F=u g_{6, \rho}, 0 \leq \rho \leq 6$, then by choosing $h$ and $N$ as in (2.18) and (2.19) the following estimate holds:

$$
\left|\left\langle u^{(6)}, S_{k}\right\rangle-h \sum_{j=-M}^{N} \sum_{i=0}^{6} \frac{u\left(x_{j}\right)}{\phi^{\prime}\left(x_{j}\right) h^{i}} \delta_{k j}^{(i)} g_{6, i}\right| \leq \nu_{6} M^{3} e^{\frac{-\pi d}{h}},
$$

where $\nu_{6}$ depends on $u, w, \phi$ and $d$.

Proof. (i) We shall only prove the case $i=6$ since the proof of the remaining cases is similar. Using the identity in (2.15) for the domain $D_{E}$ which says

$$
|K(\phi, h)(z)|_{z \in \partial D_{E}}=e^{-\pi d / h},
$$

along with the aid of the inequalities in (2.70), we obtain the bound

$$
\begin{aligned}
\left|I_{F}^{(6)}\right|=\mid\left\langle u^{(6)}, S_{k}\right\rangle & -h \sum_{j=-\infty}^{\infty} \frac{u\left(x_{j}\right)}{\phi^{\prime}\left(x_{j}\right)}\left(S_{k} w\right)^{(6)}\left(x_{j}\right) \mid \\
\leq & \frac{e^{\frac{-\pi d}{h}}}{2}\left\{\sum_{i=0}^{6} \int_{\partial D_{E}}|u(z)|\left|g_{6, i}(x)\right|\left|\frac{\frac{d^{(i)}}{d \phi^{(i)}} S(k, h) \circ \phi(z)}{\sin \left(\frac{\pi \phi(z)}{h}\right)}\right||d z|\right\} .
\end{aligned}
$$

Thus, recalling the definintion of $T(f)$ given by (2.12), we have

$$
\left|I_{F}^{(6)}\right| \leq\left[\sum_{i=0}^{6} C_{i}(h, d) T\left(u g_{6, i}\right)\right] e^{-\pi d / h} \equiv C_{6} e^{-\pi d / h} .
$$


(ii) From (2.21)-(2.25) we have the inequalities

$$
\begin{aligned}
& \left|\delta_{k j}^{(6)}\right| \leq \frac{\pi^{6}}{7}, \quad\left|\delta_{k j}^{(5)}\right| \leq \frac{2 \pi^{4}-10 \pi^{2}+15}{4}, \quad\left|\delta_{k j}^{(4)}\right| \leq \frac{\pi^{4}}{5} \\
& \left|\delta_{k j}^{(3)}\right| \leq \frac{2 \pi^{2}-3}{4}, \quad\left|\delta_{k j}^{(2)}\right| \leq \frac{\pi^{2}}{3}, \quad\left|\delta_{k j}^{(\nu)}\right| \leq 1, \quad \nu=1,2 .
\end{aligned}
$$

We also have

$$
\begin{aligned}
& \left|\left\langle u^{(6)}, S_{k}\right\rangle-h \sum_{j=-M}^{N} \sum_{i=0}^{6} \frac{u\left(x_{j}\right)}{\phi^{\prime}\left(x_{j}\right) h^{i}} \delta_{k j}^{(i)} g_{6, i}\right| \\
& \leq\left|I_{F}^{(6)}\right|+\sum_{i=1}^{6}\left[\left|h \sum_{j=-\infty}^{-M-1} \frac{u\left(x_{j}\right)}{\phi^{\prime}\left(x_{j}\right)} g_{6, i} \frac{\delta_{k j}^{(i)}}{h^{i}}\right|+\left|h \sum_{j=N+1}^{\infty} \frac{u\left(x_{j}\right)}{\phi^{\prime}\left(x_{j}\right)} g_{6, i} \frac{\delta_{k j}^{(i)}}{h^{i}}\right|\right] .
\end{aligned}
$$

But

$$
\begin{aligned}
\left|h \sum_{j=-\infty}^{-M-1} \frac{u\left(x_{j}\right)}{\phi^{\prime}\left(x_{j}\right)} g_{6,6} \frac{\delta_{k j}^{(6)}}{h^{6}}\right| & \leq h \sum_{j=M+1}^{\infty}\left|\frac{u}{\phi^{\prime}} g_{6,6}\left(x_{-j}\right)\right|\left|\frac{\delta_{k,-j}^{(6)}}{h^{6}}\right| \\
& \leq \frac{\nu \pi^{6}}{7 h^{5}} \sum_{j=M+1}^{\infty} e^{-\alpha j h} \equiv \frac{\nu \pi^{6}}{7 \alpha h^{6}} e^{-\alpha M h}
\end{aligned}
$$

and in exactly the same fashion

$$
\begin{aligned}
\left|h \sum_{j=N+1}^{\infty} \frac{u\left(x_{j}\right)}{\phi^{\prime}\left(x_{j}\right)} g_{6,6} \frac{\delta_{k j}^{(6)}}{h^{6}}\right| & \leq h \sum_{j=N+1}^{\infty}\left|\frac{u}{\phi^{\prime}} g_{6,6}\left(x_{j}\right)\right|\left|\frac{\delta_{k, j}^{(6)}}{h^{6}}\right| \\
& \leq \frac{\nu \pi^{6}}{7 h^{5}} \sum_{j=N+1}^{\infty} e^{-\beta j h} \equiv \frac{\nu \pi^{6}}{7 \beta h^{6}} e^{-\beta N h} .
\end{aligned}
$$

The remaining terms in equation (2.76) are bounded in a similar manner so that (2.76) takes the form

$$
\begin{aligned}
\left|\left\langle u^{(6)}, S_{k}\right\rangle-h \sum_{j=-M}^{N} \sum_{i=0}^{6} \frac{u\left(x_{j}\right)}{\phi^{\prime}\left(x_{j}\right) h^{i}} \delta_{k j}^{(i)} g_{6, i}\right| \\
\leq \nu \\
\quad\left[\frac{\pi^{6}}{7 h^{6}}+\frac{2 \pi^{4}-10 \pi^{2}+15}{4 h^{5}}+\frac{\pi^{4}}{5 h^{4}}\right. \\
\left.+\frac{2 \pi^{2}-3}{4 h^{3}}+\frac{\pi^{2}}{3 h^{2}}+\frac{1}{h}\right]\left(\frac{e^{-\alpha M h}}{\alpha}+\frac{e^{-\beta N h}}{\beta}\right)+C_{6} e^{\frac{-\pi d}{h}} .
\end{aligned}
$$

From Theorem 4.2 in [9], for suffciently small $h$, there exist constants $k_{\sigma}$ such that

$$
C_{\sigma}(h, d) \leq k_{\sigma} h^{1-\sigma}, \quad 0 \leq \sigma \leq 6
$$


Substituting this and the selections (2.18) and (2.19) into the right-hand side of equation (2.79) leads to (2.74), where

$$
\begin{aligned}
\nu_{6} \equiv \nu\left[\frac{\pi^{6}}{7}\left(\frac{\alpha}{\pi d}\right)^{3}\right. & +\left(\frac{2 \pi^{4}-10 \pi^{2}+15}{4}\right)\left(\frac{\alpha}{\pi d}\right)^{5 / 2} \\
& +\frac{\pi^{4}}{5}\left(\frac{\alpha}{\pi d}\right)^{2}+\left(\frac{2 \pi^{2}-3}{4}\right)\left(\frac{\alpha}{\pi d}\right)^{3 / 2} \\
& \left.+\frac{\pi^{2}}{3}\left(\frac{\alpha}{\pi d}\right)+\left(\frac{\alpha}{\pi d}\right)^{1 / 2}+\sqrt{\frac{\alpha}{\pi d}}\right]\left(\frac{1}{\alpha}+\frac{1}{\beta}\right)+k_{6} .
\end{aligned}
$$

The remaining inner product approximations are listed in the following theorem which extends Theorem 3.1 in [12].

Theorem 2.4. Let $\phi$ be as before, $x_{j}=\phi^{-1}(j h)$ and $T($.$) be defined by (2.12).$ Let $C_{j}(h, d)$ have the same meaning as in (2.70), and let $C_{j}$ be given by (2.71) (2.72), $0 \leq j \leq 6$. If $f w, u p_{0} w, u g_{i, j}$ are in $B\left(D_{E}\right)$ and if $u g_{i, j}$ decays exponentially with respect to $\phi$, for $0 \leq i \leq 6,0 \leq j \leq i$, then by choosing $h$ and $N$ as in (2.18) and (2.19), respectively, we have the following bounds:

$$
\begin{gathered}
\left|\left\langle f, S_{k}\right\rangle-h \frac{f\left(x_{k}\right) w\left(x_{k}\right)}{\phi^{\prime}\left(x_{k}\right)}\right| \leq \frac{1}{2} \nu_{0} T(f w) M^{-1 / 2} e^{-(\pi \alpha d M)^{1 / 2}}, \\
\left|\left\langle p_{0} u, S_{k}\right\rangle-h \frac{p_{0}\left(x_{k}\right) u\left(x_{k}\right) w\left(x_{k}\right)}{\phi^{\prime}\left(x_{k}\right)}\right| \leq \frac{1}{2} \nu_{0} T(u v w) M^{-1 / 2} e^{-(\pi \alpha d M)^{1 / 2}}, \\
\left|\left\langle p_{1} u^{(1)}, S_{k}\right\rangle+h \sum_{j=-M}^{N} \sum_{i=0}^{1} \frac{u\left(x_{j}\right)}{\phi^{\prime}\left(x_{j}\right) h^{i}} \delta_{k j}^{(i)} g_{1, i}\right| \leq \nu_{1} M^{1 / 2} e^{-(\alpha \pi d M)^{1 / 2},} \\
\left|\left\langle p_{2} u^{(2)}, S_{k}\right\rangle-h \sum_{j=-M}^{N} \sum_{i=0}^{2} \frac{u\left(x_{j}\right)}{\phi^{\prime}\left(x_{j}\right) h^{i}} \delta_{k j}^{(i)} g_{2, i}\right| \leq \nu_{2} M e^{-(\alpha \pi d M)^{1 / 2}}, \\
\left|\left\langle p_{3} u^{(3)}, S_{k}\right\rangle+h \sum_{j=-M}^{N} \sum_{i=0}^{3} \frac{u\left(x_{j}\right)}{\phi^{\prime}\left(x_{j}\right) h^{i}} \delta_{k j}^{(i)} g_{3, i}\right| \leq \nu_{3} M^{3 / 2} e^{-(\alpha \pi d M)^{1 / 2}}, \\
\left|\left\langle p_{4} u^{(4)}, S_{k}\right\rangle-h \sum_{j=-M}^{N} \sum_{i=0}^{4} \frac{u\left(x_{j}\right)}{\phi^{\prime}\left(x_{j}\right) h^{i}} \delta_{k j}^{(i)} g_{4, i}\right| \leq \nu_{4} M^{2} e^{-(\alpha \pi d M)^{1 / 2}}, \\
\left|\left\langle p_{5} u^{(5)}, S_{k}\right\rangle+h \sum_{j=-M}^{N} \sum_{i=0}^{5} \frac{u\left(x_{j}\right)}{\phi^{\prime}\left(x_{j}\right) h^{i}} \delta_{k j}^{(i)} g_{5, i}\right| \leq \nu_{5} M^{5 / 2} e^{-(\alpha \pi d M)^{1 / 2}},
\end{gathered}
$$

and finally

$$
\left|\left\langle u^{(6)}, S_{k}\right\rangle-h \sum_{j=-M}^{N} \sum_{i=0}^{6} \frac{u\left(x_{j}\right)}{\phi^{\prime}\left(x_{j}\right) h^{i}} \delta_{k j}^{(i)} g_{6, i}\right| \leq \nu_{6} M^{3} e^{-(\alpha \pi d M)^{1 / 2}} .
$$

Theorem 2.4 contains all the approximations needed to formulate the discrete Sinc-Galerkin system for problem (1.1)-(1.2). Replacing each term of (2.9) with the approximations defined in (2.82)-(2.89) and replacing $u\left(x_{j}\right)$ by $c_{j}$ and dividing by $h$, we obtain the following theorem. 
Theorem 2.5. If the assumed approximate solution of the boundary-value problem (1.1)-(1.2) is (2.5), then the discrete Sinc-Galerkin system for the determination of the unknown coefficients $\left\{c_{j}\right\}_{j=-M}^{N}$ is given by

$$
\begin{array}{r}
\sum_{j=-M}^{N}\left\{\sum_{i=0}^{6} \frac{1}{h^{i}} \delta_{k j}^{(i)} \frac{g_{6, i}\left(x_{j}\right)}{\phi^{\prime}\left(x_{j}\right)} c_{j}-\sum_{i=0}^{5} \frac{1}{h^{i}} \delta_{k j}^{(i)} \frac{g_{5, i}\left(x_{j}\right)}{\phi^{\prime}\left(x_{j}\right)} c_{j}+\sum_{i=0}^{4} \frac{1}{h^{i}} \delta_{k j}^{(i)} \frac{g_{4, i}\left(x_{j}\right)}{\phi^{\prime}\left(x_{j}\right)} c_{j}\right. \\
\left.-\sum_{i=0}^{3} \frac{1}{h^{i}} \delta_{k j}^{(i)} \frac{g_{3, i}\left(x_{j}\right)}{\phi^{\prime}\left(x_{j}\right)} c_{j}+\sum_{i=0}^{2} \frac{1}{h^{i}} \delta_{k j}^{(i)} \frac{g_{2, i}\left(x_{j}\right)}{\phi^{\prime}\left(x_{j}\right)} c_{j}-\sum_{i=0}^{1} \frac{1}{h^{i}} \delta_{k j}^{(i)} \frac{g_{1, i}\left(x_{j}\right)}{\phi^{\prime}\left(x_{j}\right)} c_{j}\right\} \\
+\frac{p_{0}\left(x_{k}\right) w\left(x_{k}\right)}{\phi^{\prime}\left(x_{k}\right)} c_{k}=\frac{f\left(x_{k}\right) w\left(x_{k}\right)}{\phi^{\prime}\left(x_{k}\right)}
\end{array}
$$

$k=-M, \ldots, N$.

Proof. Combine Lemma 2.3 and Theorem 2.4.

To obtain a matrix representation of the equations in (2.90), denote by $\mathbf{I}^{(i)}$, $1 \leq i \leq 6$, the $m \times m$ matrices $(m=M+N+1)$ whose $j k$-th entry is given by (2.21) -(2.27), respectively. Let $\mathbf{D}\left(g\left(x_{j}\right)\right)$ denote the $m \times m$ diagonal matrix with diagonal entries $g\left(x_{j}\right), j=-M,-M+1, \ldots, 0, \ldots, N$. Let $\mathbf{c}$ be the $m$-vectors with $j$-th component given by $c_{j}$ and let $\mathbf{1}$ be the $m$-vector each of whose components is 1 . In this notation the system in (2.90) takes the matrix form

$$
\mathbf{A} \mathbf{c}=\mathbf{D}\left(\frac{w f}{\phi^{\prime}}\right) \mathbf{1}
$$

where

$$
\mathbf{A}=\sum_{i=0}^{6} \frac{1}{h^{i}} \mathbf{I}^{(i)} \mathbf{D}\left(a_{i}\right),
$$

and the functions $a_{j}(x), 0 \leq j \leq 6$, are given by

$$
\begin{gathered}
a_{0}=\left(g_{6,0}-g_{5,0}+g_{4,0}-g_{3,0}+g_{2,0}-g_{1,0}+p_{0} w\right) / \phi^{\prime}, \\
a_{1}=\left(g_{6,1}-g_{5,1}+g_{4,1}-g_{3,1}+g_{2,1}-g_{1,1}\right) / \phi^{\prime}, \\
a_{2}=\left(g_{6,2}-g_{5,2}+g_{4,2}-g_{3,2}+g_{2,2}\right) / \phi^{\prime}, \\
a_{3}=\left(g_{6,3}-g_{5,3}+g_{4,3}-g_{3,3}\right) / \phi^{\prime}, \\
a_{4}=\left(g_{6,4}-g_{5,4}+g_{4,4}\right) / \phi^{\prime}, \\
a_{5}=\left(g_{6,5}-g_{5,5}\right) / \phi^{\prime},
\end{gathered}
$$

and

$$
a_{6}=\left(g_{6,6}\right) / \phi^{\prime} .
$$

Note that the matrices $\mathbf{I}^{(2)}, \mathbf{I}^{(4)}$ and $\mathbf{I}^{(6)}$ are $m \times m$ symmetric matrices and the matrices $\mathbf{I}^{(1)}, \mathbf{I}^{(3)}$ and $\mathbf{I}^{(5)}$ are $m \times m$ skew-symmetric matrices [7]. The matrix $\mathbf{I}^{(0)}$ is the $m \times m$ identity matrix.

Now we have a linear system of $m$ equations of the $m$ unknown coefficients, namely, $\left\{c_{j}, j=-M, \ldots, N\right\}$. We can obtain the coefficients of the approximate solution by solving this linear system. This system (2.91) may be easily solved by a variety of methods. In this paper we use the $Q-R$ method [6, 11]. The solution 
$\mathbf{c}=\left(c_{-M}, c_{-M+1}, \ldots, c_{N}\right)^{T}$ gives the coefficients in the approximate Sinc-Galerkin $u_{m}(x)$ of $u(x)$.

\section{TREATMENT OF THE BOUNDARY CONDITIONS}

In the previous section the development of the Sinc-Galerkin technique for homogeneous boundary conditions provided a practical approach since the Sinc functions composed with various conformal mappings, $S(j, h) \circ \phi$, are zero at the endpoints of the interval. If the boundary conditions are nonhomogeneous, then these conditions need be converted to homogeneous conditions via an interpolation by a known function. For example, consider

$$
L u(x)=u^{(6)}+\sum_{j=0}^{5} p_{j}(x) u^{(j)}=f(x), \quad 0 \leq x \leq 1,
$$

subject to boundary conditions

$$
u^{(i)}(0)=\Theta_{i}, \quad u^{(i)}(1)=\Phi_{i}, \quad i=0,1,2 .
$$

The nonhomogeneous boundary conditions in (3.2) can be transformed to homogeneous boundary conditions by the change of dependent variable

$$
\Psi(x)=u(x)-\Lambda(x),
$$

where $\Lambda(x)$ is the interpolating polynomial that satisfies $\Lambda^{(i)}(0)=\Theta_{i}$ and $\Lambda^{(i)}(1)=$ $\Phi_{i}, i=0,1,2$. It is easy to see that

$$
\Lambda(x)=\sum_{i=0}^{5} \rho_{i} x^{i}
$$

and

$$
\begin{gathered}
\rho_{0}=\Theta_{0}, \quad \rho_{1}=\Theta_{1}, \quad \rho_{2}=\frac{\Theta_{2}}{2}, \\
\rho_{3}=\frac{1}{2}\left[\left(20 \Phi_{0}-8 \Phi_{1}+\Phi_{2}\right)-\left(20 \Theta_{0}+12 \Theta_{1}+3 \Theta_{2}\right)\right], \\
\rho_{4}=\left[\left(-15 \Phi_{0}+7 \Phi_{1}-\Phi_{2}\right)+\left(15 \Theta_{0}+8 \Theta_{1}+\frac{3}{2} \Theta_{2}\right)\right], \\
\rho_{5}=\frac{1}{2}\left[\left(12 \Phi_{0}-6 \Phi_{1}+\Phi_{2}\right)-\left(12 \Theta_{0}+6 \Theta_{1}+\Theta_{2}\right)\right] .
\end{gathered}
$$

The new problem with homogeneous boundary conditions is then

$$
L \Psi(x)=\Psi^{(6)}(x)+\sum_{j=0}^{5} p_{j}(x) \Psi^{(j)}(x)=\tilde{f}(x), \quad 0 \leq x \leq 1,
$$

subject to the boundary conditions

$$
\Psi^{(i)}(0)=0, \quad \Psi^{(i)}(1)=0, \quad i=0,1,2
$$

where

$$
\begin{aligned}
\tilde{f}(x) & =f(x)-L \Lambda(x) \\
& =f(x)-\sum_{j=0}^{5} p_{j}(x) \Lambda^{(j)}(x) .
\end{aligned}
$$


Now apply the standard Sinc-Galerkin method to (3.5). The resulting discrete system can be written as

$$
\mathbf{A} \mathbf{c}=\mathbf{D}\left(\frac{\tilde{f} w}{\phi^{\prime}}\right) \mathbf{1}
$$

where the $m \times m$ matrix $A$ is formed as

$$
\mathbf{A}=\sum_{i=0}^{6} \frac{1}{h^{i}} \mathbf{I}^{(i)} \mathbf{D}\left(a_{i}\right),
$$

where $a_{i}, 0 \leq i \leq 6$, are defined by (2.93)-(2.99), respectively. Here, we define an approximate solution of (3.5) via the formula

$$
\Psi_{m}(x)=\sum_{j=-M}^{N} c_{j} S_{j}(x), \quad m=M+N+1 .
$$

Then the approximate solution of (3.1) is

$$
u_{m}(x)=\sum_{j=-M}^{N} c_{j} S_{j}(x)+\Lambda(x),
$$

where

$$
\begin{aligned}
\Lambda(x)= & \Theta_{0}+\Theta_{1} x+\left(\frac{\Theta_{2}}{2}\right) x^{2} \\
& +\frac{1}{2}\left[\left(20 \Phi_{0}-8 \Phi_{1}+\Phi_{2}\right)-\left(20 \Theta_{0}+12 \Theta_{1}+3 \Theta_{2}\right)\right] x^{3} \\
& +\left[\left(-15 \Phi_{0}+7 \Phi_{1}-\Phi_{2}\right)+\left(15 \Theta_{0}+8 \Theta_{1}+\frac{3}{2} \Theta_{2}\right)\right] x^{4} \\
& +\frac{1}{2}\left[\left(12 \Phi_{0}-6 \Phi_{1}+\Phi_{2}\right)-\left(12 \Theta_{0}+6 \Theta_{1}+\Theta_{2}\right)\right] x^{5} .
\end{aligned}
$$

Typical choices of the weight function are given by (2.8). The resulting discrete system for the coefficients $\mathbf{c}=\left(c_{-M}, \ldots, c_{N}\right)^{T}$ in the approximate Sinc solution (3.10) is exactly the system in (2.91), with $f$ replaced by $\tilde{f}$. Notice that if $\Theta_{i}=\Phi_{i}=0, i=0,1,2$, then the discrete system obtained (3.8) and the assumed solution (3.10) reduce to (2.91) and (2.5), respectively.

\section{NumERICAL EXAMPLES}

The three examples included in this section were selected in order to illustrate the performance of the Sinc-Galerkin method in solving sixth order boundary-value problems. In the first example, the boundary conditions are homogeneous. For this case, the Sinc-Galerkin method can be applied to the problem without any modification and the discrete Sinc system defined by (2.91) is used to compute the coefficients $\left\{c_{j}, j=-M, \ldots, N\right\}$ in (2.5).

The next two examples demonstrate that the Sinc-Galerkin method can be applied to solve nonhomogeneous boundary conditions. In each of the two examples, the discrete Sinc system defined by (3.8) is used to compute the coefficients $\left\{c_{j}, j=-M, \ldots, N\right\}$ in (3.11).

We also compare our method with the modified decomposition method introduced in [17]. It is shown that the Sinc-Galerkin method yields better results. 
All examples are approximated using the selection

and

$$
h=\sqrt{\frac{\pi d}{\alpha M}},
$$

$$
N=\left[\left|\frac{\alpha}{\beta} M+1\right|\right]
$$

Note that if $\frac{\alpha}{\beta} M$ is an integer, it suffices to choose $N=\frac{\alpha}{\beta} M$, and the angle $d$ is taken to be $\frac{\pi}{2}$.

Example 4.1. Consider the boundary-value problem

$$
u^{(6)}(x)+e^{-x} u=-720+\left(x-x^{2}\right)^{3} e^{-x}, \quad 0 \leq x \leq 1,
$$

subject to the boundary conditions

$$
u^{(i)}(0)=0, \quad u^{(i)}(1)=0, \quad i=0,1,2,
$$

which has the solution given by

$$
u(x)=x^{3}(1-x)^{3} .
$$

The solution $u$ to the problem (4.1)-(4.3) is approximated by

$$
u_{m}(x)=\sum_{j=-M}^{N} c_{j} S_{j}, \quad m=M+N+1 .
$$

By using the approximations (2.82)-(2.89), we get a linear system of order $m$ for the $c_{j}$. The system takes the form

$$
\mathbf{A c}=\mathbf{D}\left(\frac{f w}{\phi^{\prime}}\right) \mathbf{1}
$$

where $A$ is given by

$$
\mathbf{A}=\sum_{i=1}^{6} \frac{1}{h^{i}} \mathbf{I}^{(i)} \mathbf{D}\left(\frac{g_{6, i}}{\phi^{\prime}}\right)+\mathbf{D}\left(\frac{g_{6,0}+p_{0} w}{\phi^{\prime}}\right) .
$$

The parameters $M=N=32$ and $\alpha=\beta=\frac{3}{2}$ are used. The approximate and exact solutions are displayed in Table 4.1. We use the relative error, defined as

$$
\text { Absolute Relative Error (ARE) }=\frac{\left|u_{\text {exact solution }}-u_{\text {Sinc-Galerkin }}\right|}{\left|u_{\text {exact solution }}\right|}
$$

Example 4.2. For the sake of comparison only, we consider the same problem discussed by Wazwaz [17], who used the modified decomposition method to obtain his numerical solution. Consider the boundary-value problem

$$
u^{(6)}-u=-6 \exp (x), \quad 0 \leq x \leq 1,
$$

subject to the boundary conditions

$$
\begin{array}{lll}
u(0)=1, & u^{\prime}(0)=0, & u^{\prime \prime}(0)=-1, \\
u(1)=0, & u^{\prime}(1)=-e, & u^{\prime \prime}(1)=-2 e
\end{array}
$$

which has the solution

$$
u(x)=(1-x) \exp (x)
$$


TABLE 4.1.

\begin{tabular}{|r|l|l|l|}
\hline $\mathrm{x}$ & Exact Solution & Sinc-Galerkin & ARE $1.0 e^{-3}$ \\
\hline 0.0 & 0.0 & 0.0 & - \\
\hline 0.1675 & 0.002711 & 0.002710 & 0.45 \\
\hline 0.2764 & 0.008000 & 0.008997 & 0.32 \\
\hline 0.3449 & 0.011534 & 0.011531 & 0.28 \\
\hline 0.4205 & 0.014469 & 0.014465 & 0.26 \\
\hline 0.5 & 0.015625 & 0.015620 & 0.25 \\
\hline 0.6550 & 0.011539 & 0.011536 & 0.28 \\
\hline 0.7828 & 0.004915 & 0.004913 & 0.37 \\
\hline 0.8324 & 0.002715 & 0.002714 & 0.45 \\
\hline 0.9041 & 0.000651 & 0.000651 & 0.73 \\
\hline 1.0 & 0.00 & 0.00 & - \\
\hline
\end{tabular}

TABLE 4.2 .

\begin{tabular}{|r|l|l|l|}
\hline $\mathrm{x}$ & Exact Solution & Sinc-Galerkin & $\mathrm{ARE} 1.0 e^{-3}$ \\
\hline 0.0 & 1.0 & 1.0 & 0.0 \\
\hline 0.0414 & 0.99911 & 0.99911 & 0.00 \\
\hline 0.0865 & 0.99603 & 0.99603 & 0.00 \\
\hline 0.1721 & 0.98337 & 0.98337 & 0.00 \\
\hline 0.3131 & 0.93944 & 0.93943 & 0.01 \\
\hline 0.5 & 0.82436 & 0.82432 & 0.03 \\
\hline 0.6868 & 0.62243 & 0.62239 & 0.07 \\
\hline 0.8278 & 0.39404 & 0.39400 & 0.09 \\
\hline 0.9134 & 0.21587 & 0.21585 & 0.10 \\
\hline 0.9585 & 0.10822 & 0.10821 & 0.10 \\
\hline 1.0 & 0.00 & 0.00 & 0.0 \\
\hline
\end{tabular}

TABLE 4.3. Maximum Absolute Relative Error (MARE)

\begin{tabular}{|l|l|}
\hline Sinc-Galerkin method & The decomposition method [17] \\
\hline 0.1 E-3 & 1.77 E-3 \\
\hline
\end{tabular}

The selected parameters $\alpha=\beta=\frac{1}{2}, M=N=16$, yield $h=\frac{\pi}{4}$. Table 4.2 exhibits a comparison between the errors obtained by using the Sinc-Galerkin method and the modified decomposition method of [17]. The Maximum Absolute Relative Errors (MARE) are tabulated in Table 4.3.

Example 4.3. Consider the boundary-value problem

$$
u^{(6)}+u^{(3)}+u^{(2)}-u=\exp (-x)\left(-15 x^{2}+78 x-114\right), \quad 0 \leq x \leq 1,
$$

subject to the boundary conditions

$$
\begin{array}{lll}
u(0)=0, & u^{\prime}(0)=0, & u^{\prime \prime}(0)=0, \\
u(1)=1 / e, & u^{\prime}(1)=2 / e, & u^{\prime \prime}(1)=1 / e,
\end{array}
$$


TABLE 4.4.

\begin{tabular}{|r|l|l|l|}
\hline $\mathrm{x}$ & Exact Solution & Sinc-Galerkin & ARE $1.0 e^{-3}$ \\
\hline 0.0 & 0.0 & 0.0 & - \\
\hline 0.0414 & 0.00006 & 0.00006 & 0.0 \\
\hline 0.0865 & 0.00059 & 0.00059 & 0.0 \\
\hline 0.1721 & 0.00429 & 0.00429 & 0.0 \\
\hline 0.3131 & 0.02244 & 0.02244 & 0.0 \\
\hline 0.5 & 0.07581 & 0.07582 & 0.08 \\
\hline 0.6868 & 0.16301 & 0.16302 & 0.1 \\
\hline 0.8278 & 0.24789 & 0.24792 & 0.1 \\
\hline 0.9134 & 0.30570 & 0.30573 & 0.1 \\
\hline 0.9585 & 0.33767 & 0.33771 & 0.1 \\
\hline 1.0 & 0.36787 & 0.36787 & 0.0 \\
\hline
\end{tabular}

which has the exact solution

$$
u(x)=x^{3} \exp (-x) .
$$

The parameters $M=N=16$ and $\alpha=\beta=\frac{1}{2}$ are used. Table 4.4 exhibits the exact and numerical solution and the relative errors.

From the above examples, we can see that the accuracy is good even when $M$ is small. These examples show that our method is efficient to deal with problem (1.1)-(1.2). All computations associated with the above examples were performed by using MATLAB. Thus, the Sinc-Galerkin method is a useful numerical tool for solving sixth order boundary-value problem.

\section{REFERENCES}

1. R. Agarwal, Boundary Value Problems for Higher Ordinary Differential Equations, World Scientific, Singapore, 1986. MR 90j:34025

2. P. Baldwin, Asymptotic estimates of the eigenvalues of a sixth order boundary value problem obtained by using global phase-integral methods, Phil. Trans. Roy. Soc. London A, 322 (1987) 281-305. MR 88g:76035

3. A. Boutayeb and E. Twizell, Numerical methods for the solution of special sixth-order boundary value problems, Int. J. Comput. Math., 45 (1992) 207-233.

4. M. Chawla and C. Katti, Finite difference methods for two-point boundary value problems involving higher order differential equations, BIT, 19 (1979) 27-33. MR 80h:65055

5. K. El-Kamel, Sinc numerical solution for solitons and solitary waves, J. Comput. Appl. Math., 130 (2001), 283-292. MR 2002c:65170

6. G. H. Golub and C. F. Vanloan, Matrix Computations, Third Ed. The Johns Hopkins Press Ltd., London, 1996.

7. V. Grenander and G. Szego, Toeplitz Forms and Their Applications, Second Ed. Chelsea Publishing Co., Orlando, 1985. MR 97g:65006

8. J. Lund, Symmetrization of the Sinc-Galerkin method for boundary value problems, Math. Comp., 47 (1986), 571-588. MR 87m:65186

9. J. Lund and K. Bowers, Sinc Methods for Quadrature and Differential Equations, SIAM, Philadelphia, PA, 1992. MR 93i:65004

10. K. Michael, Fast iterative methods for symmetric Sinc-Galerkin system, IMA J. Numer. Anal., 19 (1999), 357-373. MR 2001b:65083

11. E. Part-Enander, A. Sjoberg, B. Melin and P. Isaksson, The Matlab Handbook, Addison Wesley Longman, 1996.

12. R. C. Smith et al., The Sinc-Galerkin method for fourth-order differential equations, SIAM J. Numer. Anal., 28 (1991), 760-788. MR 92c:65096 
13. S. Siddiqi and E. Twizell, Spline solutions of linear sixth order boundary value problems, Int. J. Comput. Math., 60 (1996) 295-304.

14. F. Stenger, Numerical Methods Based on Sinc and Analytic Functions, Springer, New York, 1993. MR 94k:65003

15. F. Stenger, Summary of Sinc numerical methods, J. Compt. Appl. Math., 121 (2000) 379-420. MR 2001d:65018

16. E. Twizell and A. Boutayed, Numerical methods for the solution of special and general sixthorder boundary value problems with applications to Benard layer eigenvalue problems, Proc. Roy. Soc. London A, 431 (1990) 433-450. MR 91m:65209

17. A. Wazwaz, The numerical solution of sixth order boundary value problems by the modified decomposition method, Appl. Math. Comput., 118 (2001) 311-325. MR 2001k:65122

Department of Mathematical Sciences, Faculty of Engineering, Mansoura UniverSity, Mansoura, Egypt

E-mail address: gamel_eg@yahoo.com

Department of Mathematics, University of Central Florida, Orlando, Florida 32816

E-mail address: jcannon@pegasus.cc.ucf.edu

Department of Mathematical Sciences, DePaul University, Chicago, Illinois 60614

E-mail address: azayed@math.depaul.edu 